\title{
Transition flight modeling and robust control of a VTOL unmanned quad tilt-rotor aerial vehicle
}

\author{
Navya Thirumaleshwar Hegde ${ }^{1}$, V.I.George ${ }^{2}$, C Gurudas Nayak $^{3}$, Kamlesh Kumar ${ }^{4}$ \\ ${ }_{1,2,3}$ Department of Instrumentation and Control Engineering, Manipal Institute of Technology (MIT), \\ Manipal Academy of Higher Education, India \\ ${ }^{4}$ Department of Aeronautical and Automobile Engineering, Manipal Institute of Technology (MIT), \\ Manipal Academy of Higher Education, India
}

\begin{tabular}{l}
\hline Article Info \\
\hline Article history: \\
Received Sep 27, 2019 \\
Revised Nov 29, 2019 \\
Accepted Dec 12, 2019 \\
\hline Keywords: \\
Flight control system transition \\
Flight modelling \\
Quad tiltrotor UAV \\
UAV \\
VTOL UAV
\end{tabular}

\begin{abstract}
The development of fully autonomous Unmanned Aerial Vehicles (UAV) plays a major contribution towards reducing the risk to human life in various applications including rescue teams, border patrol, police and inspection of buildings, pipelines, coasts, and terrains. Tiltrotor hybrid UAV exhibit special application value due to its unique rotor structure. The variation in the model dynamics and aerodynamics due to the tilting rotors are the major key issues and challenges which attracted the attention of many researchers. This vehicle combines the hovering capabilities of a helicopter along with the high-speed cruise capabilities of a conventional airplane by tilting its four rotors. In the present research work, the authors attempt to model a quad tilt rotor UAV using Newton-Euler formulation. A dynamic model of the vehicle is derived mathematically for horizontal, vertical and transition flight modes. A robust H-infinity control strategy is proposed, evaluated and analyzed through simulation to control the flight dynamics of the different modes of the UAV. Simulation results shows that the tiltrotor UAV achieves transition successfully.
\end{abstract}

Copyright $₫ 2020$ Institute of Advanced Engineering and Science. All rights reserved.

\section{Corresponding Author:}

Kamlesh Kumar,

Department of Aeronautical and Automobile Engineering,

Manipal Institute of Technology (MIT),

Manipal Academy of Higher Education, Manipal-576104, India.

Email:kamlesh.kumar@manipal.edu

\section{INTRODUCTION}

Unmanned aerial vehicles (UAV or drones), can achieve the tasks which are either expensive to be carried out by pilot operated vehicles or hazardous for human life [1, 2]. In recent years, UAVs are increasing in number due to their various potential applications. UAVs can be characterized into two basic types, i.e., fixed-wing and rotary-wing UAVs. Fixed-wing UAVs, such as aircraft are driven forward by turbine jet engines or propellers and wings [3]. They offer higher cruise speed, flight mileage, and less noise compared to other aircraft. Due to this, they are extensively used in commercial and military purposes. However, these UAVs cannot perform VTOL (vertical take-off and landing). Rotary wing aerial vehicles [4], such as helicopters, are driven by propellers with nacelles in the vertical direction. They can hover at a particular location but have low endurance and cruise speed. The VTOL maneuver can be performed in many ways, some of them employing the use of tilting rotor, tilting fuselage, tilting wing [5], tailsitter, thrust vectoring, multi-propeller multifunction, flapping wing. Researchers have focused on the development of UAVs having both VTOL capabilities and a high cruising speed of fixed-wing UAV, which has increased the research in the development of tiltrotor aerial vehicles [6].

The VTOL tiltrotor UAV designed for this research (inspired by XV-15 and Osprey-22), is a quad tiltrotor with central fuselage, high wing, and counter-rotation prop rotors. The empennage comprises of a 
vertical stabilizer. The prop rotors are placed on either side of the fuselage centerline. The wings has ailerons and flaps (flaperons) to provide necessary roll movements with differential deflection. In hover mode, the wing flaperons will deflect completely downward to reduce the downward force on the wing. The presented UAV is powered by four engines which are housed in nacelles at the wing tip under each prop rotor. The nacelle rotates from $90^{\circ}$ in aircraft mode to $0^{\circ}$ mast angle in hover. The tiltrotor UAV has four propellers that produce thrust by tilting the rotors. The control variables are the tilt angles and rotation speed of the rotors. Four identical wings are placed on each of the fuselage to produce lift. The vertical tail is fixed at the back to maintain vehicle stability. If the VTOL tiltrotor UAV is in "helicopter mode", then the nacelles are made vertical with respect to the wing, thereby positioning the rotors accordingly. If the UAV is in "airplane mode", then the nacelles are kept parallel to the wing by placing rotors horizontally. The flight condition in which, the nacelles works between airplane and helicopter mode is called "transition mode". In these types of UAVs, the transition occurs by rotating the nacelles, and the prop rotors work like propellers [7].

In [8], multiple issues were witnessed in quadrotor aircraft in the presence of wind disturbances and their operation at higher speeds. A linear GH-infinity controller has been applied to a nonlinear quadrotor in the presence of uncertainties and disturbances mainly as measurement noise [9]. To compensate for uncertain nonlinear multivariable systems, an intelligent robust controller based on the H-infinity control approach had been developed [10]. A mixed robust controller which is the combination of H-infinity and Proportional-Integral-Derivative (PID) controller in the presence of wind disturbance is proposed for the UAV system [11]. In presence of sinusoidal wind disturbance, an adaptive fuzzy controller showed robust performance. By selecting proper weighting functions for a linearized robust feedback H-infinity controller, the designed system was highly robust to the uncertainties and disturbances [12]. Optimal control algorithms deal with Linear Quadratic Regulator, L1, Kalman filter and H-infinity. The limitations of optimal control algorithms include reduced robustness and system vibrations. Robust controllers are implemented to deal with uncertainties for un-modeled system parameters for UAVs. Poor tracking ability is a major limitation. Using a dynamic surface method and back-stepping controller for the Multi-Input Multi-Output system, a robust adaptive tracking control algorithm was designed with unknown external disturbances and input saturation [13]. The robust performance was is achieved using multivariate $\mathrm{H}$-infinity control, where gainscheduled law is used to convert a Linear Parameter Varying model of a HARVee tilt-wing UAV with respect to deviation in the position of the Centre of Gravity (CG) [14]. A robust PID controller having high order is proposed to improve the overall system performance with the deadbeat response using model reduction techniques [15]. A complete mathematical model of 6 degrees of freedom (DoF) of tiltrotor UAV (TRUAV) is designed with aerodynamics effects. The proposed PID controllers work well in-spite of uncertainty and improve the TRUAV flight performance and control safety. Real-time implementation of TRUAV is performed in all three flight modes [16].

The main contribution of this work is the design and evaluation of the dynamics of quad tiltrotor UAV in MATLAB/SIMULINK for all the three flight modes. Controlling this aerial vehicle is difficult due to its time-varying and non-linear dynamics. Uncertainties associated with the environment and modeling errors, demands the design of a robust controller to control the UAV. A robust H-infinity controller that addresses the robust performance and compensate the stability problem of UAV in the presence of uncertainties is designed and could be utilized in a wide range of applications.

The organization of the rest of the article is, Section 2 includes the literature that had been reviewed for this research. Section 3 gives a methodology to derive the UAV model mathematically and explains flight dynamics modeling using Newton-Euler EOM. In Section 4, an overview of the presented work is discussed along with the results, discussions, achievements, and limitations. This is followed by the conclusion in Section 5 .

\section{FLIGHT MECHANICS AND MATHEMATICAL MODELING}

This section focuses on the derivation of the mathematical model of a non-linear 6 DoF quad tiltrotor UAV in hover, transition and cruise flight modes. The complexity of the UAV motions is due to the coupling of translation and rotation motion which further effects the magnitude and direction of force and moments. UAV mathematical modeling is a complex and time-consuming process. The combination of flight dynamics and mechanics associated with hover, vertical flight, transition and forward flight is as shown in Figure $1[17]$. 


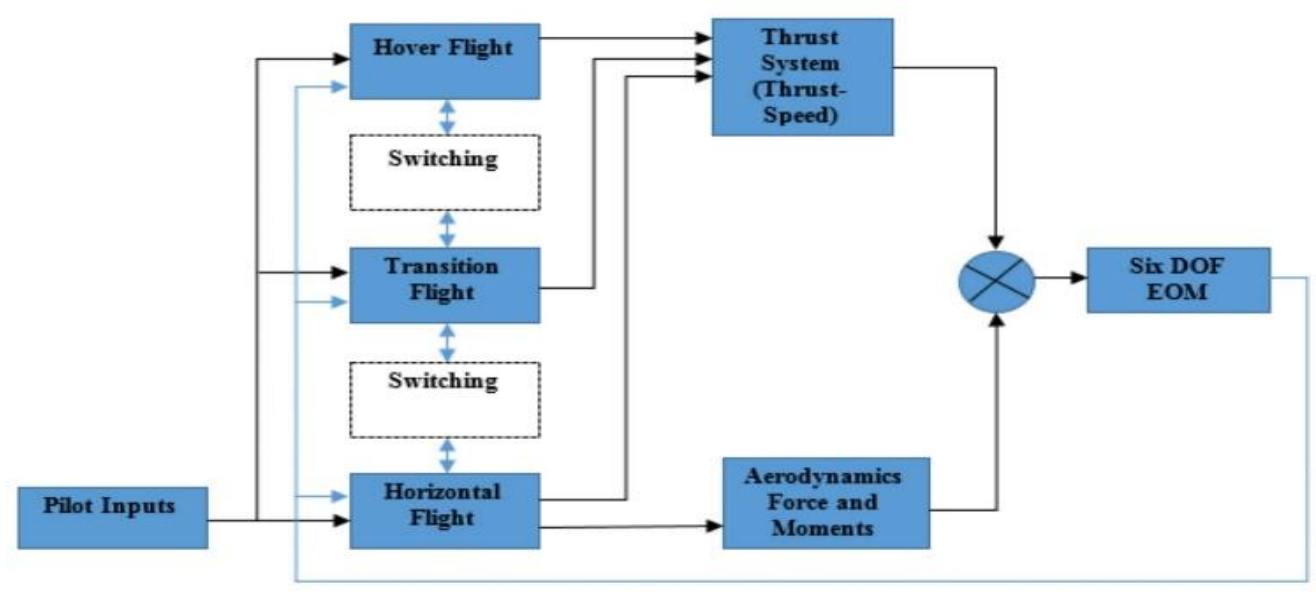

Figure 1. Block diagram representation of the dynamic model of the tiltrotor UAV

The first principle theory is used to derive the EOM of UAV. The following section presents the derivations of the aerodynamic, gravitational, thrust forces and moments [17]. The mathematical representations of the three forces are presented in (1),

$$
\begin{aligned}
& m(\dot{u}-r v+q w)+m g \sin \theta=F_{x} \quad m(\dot{v}-p w+r u)-m g \sin \phi \cos \theta=F_{y} \\
& m(\dot{w}-q u+p v)-m g \cos \phi \cos \theta=F_{z}
\end{aligned}
$$

where $p, q, r, u, v, w$ are the roll rate, pitch rate, yaw rate and velocities in the $\mathrm{x}, \mathrm{y}$ and $\mathrm{z}$-axis respectively, and $\phi, \theta, \psi$ are yaw, pitch, and roll angle, respectively. As shown in (2) gives the three moments EOM in the Body Axis System, where the response of UAV are mentioned on the left-hand side and applied moments on the right-hand side,

$$
\begin{aligned}
& \dot{p} I_{x x}-q r\left(I_{y y}-I_{z z}\right)+\left(r^{2}-q^{2}\right) I_{y z}-(\dot{r}+p q) I_{x z}+(p r-\dot{q}) I_{x y}=L \\
& \dot{q} I_{y y}-p r\left(I_{z z}-I_{x x}\right)+\left(p^{2}-r^{2}\right) I_{x z}-(\dot{p}+q r) I_{x y}+(p q-\dot{r}) I_{y z}=M \\
& \dot{r} I_{z z}-p q\left(I_{x x}-I_{y y}\right)+\left(q^{2}-p^{2}\right) I_{x y}-(q+p r) I_{y z}+(q r-\dot{p}) I_{x z}=N
\end{aligned}
$$

As shown in (3) represents the three kinematic equations,

$$
P=-\sin \theta \dot{\psi}+\dot{\phi} \quad Q=\sin \phi \cos \theta \dot{\psi}+\cos \phi \dot{\theta} \quad R=\cos \phi \cos \theta \dot{\psi}-\sin \phi \dot{\theta}
$$

where $L, M, N$ are the roll, pitch and yaw moments respectively, $I_{x}, I_{y}, I_{z}$ are the moment of inertia and $\alpha$ is the angle of attack. For trim, the sum of all force and moments acting on the body is assumed to be zero. For simplification, the UAV is symmetric about x-y plane; therefore $I_{x y}$ and $I_{y z}$ can be neglected. While deriving EOMs, many reference frames are being used to define the variables such as relative positions, forces, accelerations, inertia tensor, velocities, and aerodynamic derivatives. Every element of the UAV which contributes to forces and moments should be considered during analyses and design. The components involved for design are the wing, horizontal tail, fuselage, prop rotors and empennage with vertical tail [18].

\subsection{Linearized Model}

Using the Taylor series, the force and moments EOM is expanded about a trimmed state. By assuming small perturbation theory, perturbation products are neglected and the resulting equation can be called linear. The partial derivatives obtained are called stability derivatives, which can be split into the main variable (force/moment) and a subscript variable (perturbed control/state) [19]. Mathematical models are used to linearize a nonlinear model at trim points. Newton's laws of motion lead to a set of non-linear differential equations for the development of UAV responses, attitude, and trajectory with time. The UAV motion variables can be divided into navigation variables and aerodynamic variables. The former set of variables is used in UAV navigational computations while the latter can be used to calculate aerodynamics of the vehicle.

The derivatives of Euler angles are zero $[\dot{\phi}=\dot{\theta}=0]$ at the equilibrium point. The determination of control inputs $\left[\Omega_{1 e}, \theta_{1 e}, \Omega_{2 e}, \theta_{2 e}, \Omega_{3 e}, \theta_{3 e}, \Omega_{4 e}, \theta_{4 e}\right]$, Euler angles $\left[\theta_{e}, \phi_{e}\right]$, rotational velocities $\left[p_{e}, q_{e}, r_{e}\right]$ and 
translational velocities $\left[u_{e}, v_{e}, w_{e}\right]$ which are required to hold the UAV in equilibrium point are of major concern. The UAV motion model is developed in a nonlinear form as, $\dot{x}=F(x, u, t)$, where input variables are $u=[\mathrm{X}, \mathrm{Y}, \mathrm{Z}, \mathrm{L}, \mathrm{M}, \mathrm{N}]$ and State variables are $x=\left[\mathrm{u}, \mathrm{v}, \mathrm{w}, \mathrm{p}, \mathrm{q}, \mathrm{r}, \phi, \theta, \psi, x_{e}, y_{e}, z_{e}\right]$. After rearrangement, the derived model is represented in $(4)$, where $[v, p, r, \phi]$ are lateral-directional state variables, $[u, w, q, \theta]$ are longitudinal state variables, state vector is $y=[u, v, w, p, q, r, \phi, \theta, \psi]$, control vector $u=\left[\theta_{01}, \theta_{1 s 1}, \theta_{1 c 1}, \theta_{02}, \theta_{1 s 2}, \theta_{1 c 2}, \delta_{\text {elev }}, \delta_{\text {ail }}, \delta_{\text {rud }}\right]$. For evaluation purposes, the control vector is simplified to pilot controlsu $=\left[\theta_{0}, \delta_{\text {long }}, \delta_{\text {lat }}, \delta_{\text {ped }}\right]$.

$$
\left[\begin{array}{c}
\dot{u} \\
\dot{w} \\
\dot{q} \\
\dot{\theta} \\
\dot{v} \\
\dot{p} \\
\dot{r} \\
\dot{\phi}
\end{array}\right]=\left[\begin{array}{c}
r v-q w-g \sin \theta \\
q u-p v+g \cos \phi \cos \theta \\
p q I_{q p q}+q r I_{q q r}+p r I_{q p r}+p^{2} I_{q p^{2}}+q^{2} I_{q q^{2}}+r^{2} I_{q r^{2}} \\
q \cos \phi-r \sin \phi \\
p w-r u+g \sin \phi \cos \theta \\
p q I_{p p q}+q r I_{p q r}+p r I_{p p r}+p^{2} I_{p p^{2}}+q^{2} I_{p q^{2}}+r^{2} I_{p r^{2}} \\
p q I_{r p q}+q r I_{r q r}+p r I_{r p r}+p^{2} I_{r p^{2}}+q^{2} I_{r q^{2}}+r^{2} I_{r r^{2}} \\
p+q \sin \phi \tan \theta+r \cos \phi \tan \theta
\end{array}\right]+\left[\begin{array}{c}
X / m_{B} \\
Z / m_{B} \\
I_{X Y}^{l} L+I_{Y Y}^{l} M+I_{Y Z}^{l} N \\
0 \\
Y / m_{B} \\
I_{X X}^{l} L+I_{X Y}^{l} M+I_{X Z}^{l} N \\
I_{X Z}^{l} L+I_{Y Z}^{l} M+I_{Z Z}^{l} N \\
0
\end{array}\right]
$$

The forces and moments can be written in an approximate form by considering Taylor's theorem and theory of linear approximations (by neglecting square and products of disturbances and rates of change of perturbations with time, because of smaller magnitude). The interaction of various components of the UAV produces the force and moments and hence it is not feasible to obtain an analytical solution. As shown in (4) can be split into $A_{1}$ and $A_{2}$ as $A=A_{1}+A_{2}=\left.\frac{d F_{1}(x)}{d x}\right|_{x_{e}}+\left.\frac{d F_{2}(x, u)}{d x}\right|_{x_{e}, u_{e}}, B=\left.\frac{d F_{2}(x, u)}{d u}\right|_{x_{e}, u_{e}}$. The state and input variables are used to compute the derivatives of the solution which is further used to find $A_{2}$ and $B$ matrices.

\subsection{Transition Dynamics Modeling}

The transition is defined as the change in flight mode between horizontal and hover and vice-versa, is the most challenging part of the tiltrotor UAV flight dynamics. The tilt angle, along with which the nacelles rotate, governs the change in the total force and moments that are acting on the UAV. Since there is no forward airspeed during vertical flight mode, there is an absence of aerodynamic forces and moments being applied on the UAV. During the transition period, the horizontal force is proportional to the tilting angle of the motor. The various forces, namely, lift, drag and moments act on the UAV [20, 21] during this transition. A combination of pitching, nacelles angle and thrust that are generated in this period. The angles of the nacelles vary gradually from $90^{\circ}$ to $0^{\circ}$ and vice-versa, in predefined steps. The transition dynamics modeling can be represented in (5) and (6) [22, 23],

$$
\begin{aligned}
& m(\dot{U}-r V+q W)=-m g \sin \theta+(-D \cos \alpha+L \sin \alpha)+\left(T_{1}+T_{2}+T_{3}+T_{4}\right) \cos i_{t} \\
& m(\dot{V}-p W+r U)=F_{G_{y}}+F_{A_{y}}+F_{T_{y}} \\
& m(\dot{W}-q U+p V)=m g \cos \phi \cos \theta+(-D \sin \alpha-L \cos \alpha)-\left(T_{1}+T_{2}+T_{3}+T_{4}\right) \sin i_{t} \\
& I_{x x} \dot{p}-\left(I_{y y}-I_{z z}\right) q r-I_{x z}(\dot{r}+p q)=L_{A}+\left(T_{1}+T_{2}\right) l_{1} \sin \zeta+\left(T_{3}+T_{4}\right) l_{2} \sin \zeta \\
& I_{y y} \dot{q}-\left(I_{z z}-I_{x x}\right) p r+I_{x z}\left(p^{2}-r^{2}\right) \\
&=\left(M_{A_{f s}}+M_{A_{p}}\right)-(L \cos \alpha+D \sin \alpha)\left(X_{a c}-X_{c g}\right)+\left(T_{1}+T_{2}\right) l_{1} \sin i_{t} \cos \zeta \\
&-\left(T_{3}+T_{4}\right) l_{2} \\
& I_{z z} \dot{r}-\left(I_{x x}-I_{y y}\right) p q+I_{x z}(q r-\dot{p})=N_{A}+\left(D_{1}+D_{2}+D_{3}+D_{4}\right)
\end{aligned}
$$

where $l_{1}$ and $l_{2}$ are moment arms, $\zeta$ is the angle between $l_{1}$ and $X_{B}$ axis, $D_{1}, D_{2}, D_{3}, D_{4}$ represent the drag moments while $T_{1}, T_{2}, T_{3}, T_{4}$ indicate the thrust forces of the tiltrotor UAV. $i_{t}$ is the angle between the direction of thrust generated with respect to the horizontal axis of the rotor.

\section{CONTROL SYSTEM DESIGN AND EVALUATION FOR UAV}

Successful implementation of a UAV depends on its controllability and flying capabilities. Till date, different control methodologies have been developed for controlling UAVs ranging from classical linear and nonlinear techniques to fuzzy neural network intelligent control approaches. When it comes to navigation, guidance, and control, there is a need to make sure that the control system designed should operate optimally in all flight conditions (take-off, cruise, and landing) irrespective of atmospheric conditions. To increase the

Transition flight modeling and robust control of a VTOL unmanned quad... (Navya Thirumaleshwar Hegde) 
speed during the flight transition period, either a tiltrotor or tiltwing instead of fixed-wing can be used. Thus, designing a nonlinear control algorithm that demonstrates the high performance and robust stability in presence of significant disturbances and perturbation is a challenging problem [24]. The main objective of this work is to design a robust $\mathrm{H}$-infinity controller that tracks all types of input commands in a noisy environment. The controller is designed with a trade-off between robustness and performance such that it fulfills the described specifications.

The ultimate goal of a robust control system is to assure better system performance in the presence of model inaccuracies, noise, disturbances, and uncertainties [25]. H-infinity control system is used to achieve both performance and stability when the external Dryden turbulence is acting on the system which is very difficult to model in case of flight controller design [26]. The $\mathrm{H}$-infinity structure to control the attitude and altitude of the UAV is proposed with the design of control allocations for the transition phase. In the proposed control algorithm, the stability is enhanced by designing a rate feedback in the first loop. The reference attitude commands are obtained by giving the data from attitude to the second loop. The third loop is used to take the linear velocity back into the controller. The designed algorithm is verified for its stability and performance through thorough simulations.

Horizontal flight control system consisting of H-infinity based attitude controllers such as roll, pitch and yaw control systems in-spite of hover and transition control systems. The outputs of these controllers are the desired coordinates, i.e. the distance (height) needed to cover in order to reach the reference parameters (error values). It will be provided to the altitude controller which generates the thrust needed to reach the desired destination. The thrust will be converted to a rotor angle and is given to the motors which will provide the desired lift. The block diagram of the entire UAV flight control scheme is shown in Figure 2(a).

\section{SIMULATION RESULTS AND DISCUSSION}

Simulations are carried out on the 6DoF quad VTOL tiltrotor UAV mathematical model using Newton-Euler EOM in MATLAB/SIMULINK and the complete flight envelope (vertical take off-transitioncruise-back transition-vertical landing) is for about $12 \mathrm{sec}$. The position controller will provide desired xyz coordinates and the altitude controller will generate the required thrust for the reference altitude. The thrust signal is given to the motor to generate the required lift. The thrust and velocity signals will operate the motors and the UAV will start to function. The flowchart of the control algorithm and the entire flight simulation is explained in Figure 2(b).

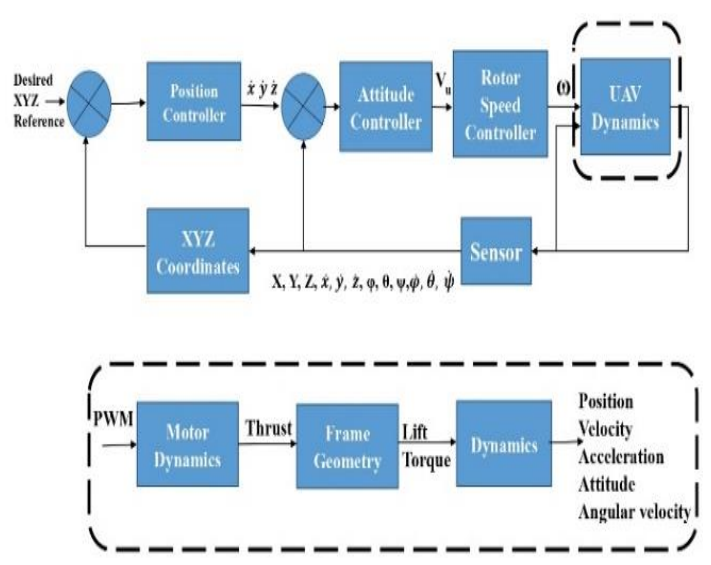

(a)

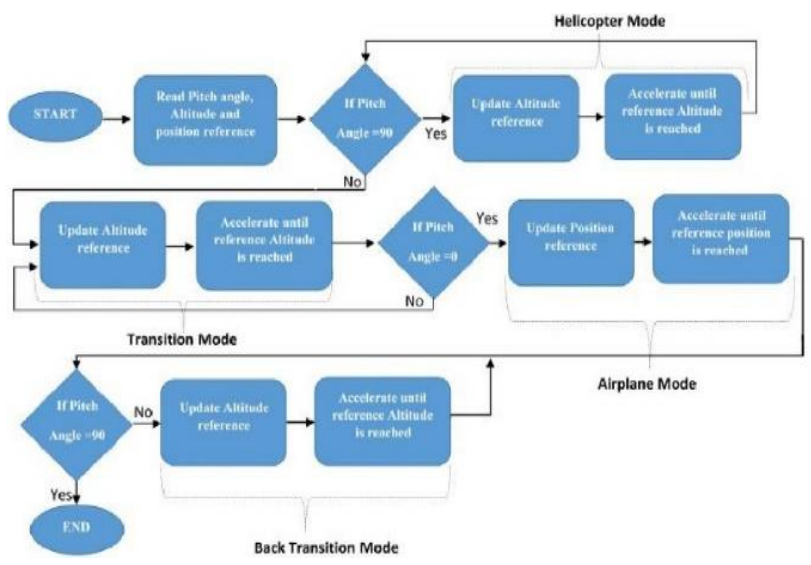

(b)

Figure 2. (a)Block diagram for the UAV flight control system, (b) The flowchart of the proposed control algorithm for quad tiltrotor UAV

The UAV starts its mode of flight when it is at rest on the ground under the influence of ground effect. It then hovers for a few instant of time and then begins the vertical take-off. During this mode, it is kept at a particular height, where the thrust produced by all the four rotors overcome its weight $(\mathrm{w}=\mathrm{mg})$ by maintaining it out of ground effect. The change of three tracking attitudes (Euler angles) and altitude responses during the flight simulation is shown in Figure 3 and Figure 4, respectively. Initially, the pitch 
angle will be $90^{\circ}$ and it is gradually reduced to increase the altitude. As the gears are driven, the four rotors are synchronously tilted and pitch towards horizontal by increasing the horizontal speed of the UAV as shown in the Figure 4.

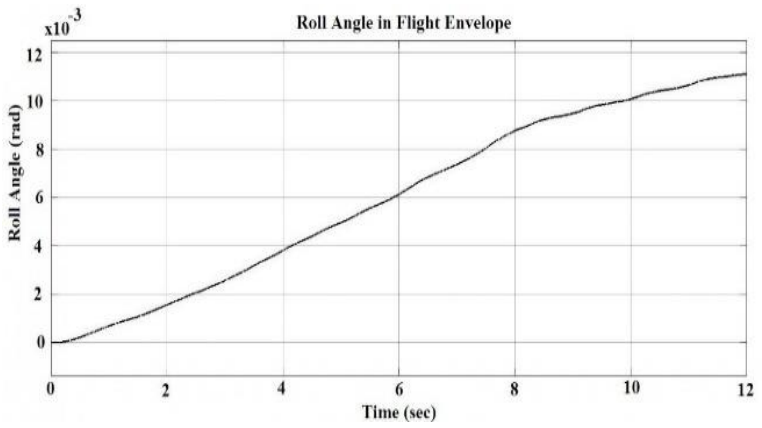

(a)

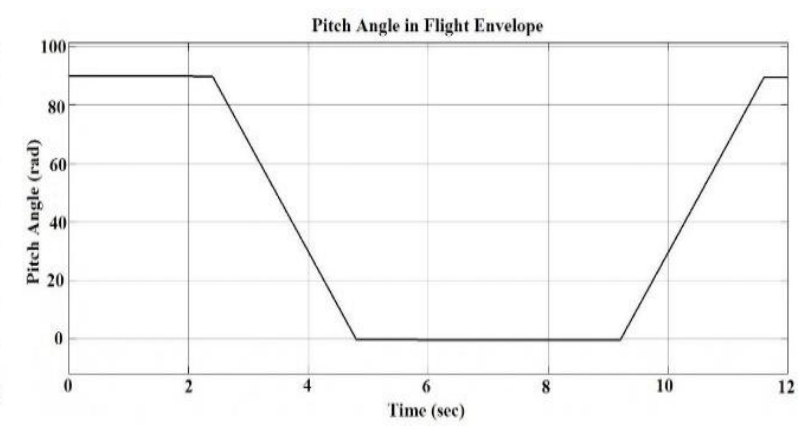

(b)

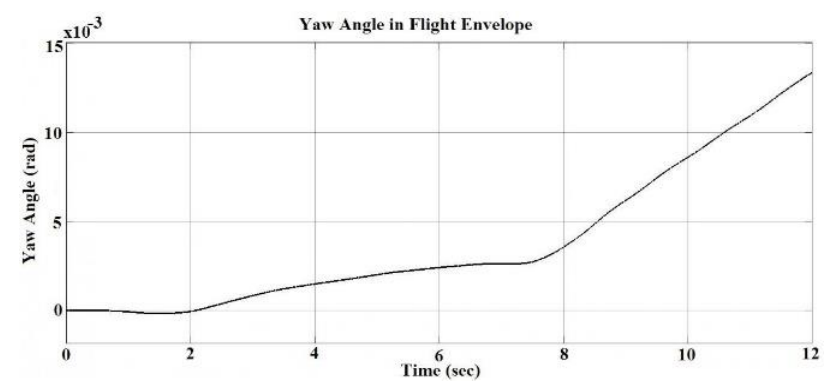

(c)

Figure 3. Euler angles change during entire flight (a) Change in Roll Angle, (b) Change in Pitch Angle, (c) Change in Yaw Angle

The attitudes are controlled by altering the blade pitch angles of the rotors to make the fixed wings to obtain a given $\alpha$ in accordance with the relative wind. During the mode of transition, the roll dynamics are regulated by the differential thrusts of the left and right rotors before the rotors tilt at a desired tilt angle. The counteractive moments control the yaw dynamics generated by the rotation of rotors. Pitch dynamics are regulated by the differential thrusts generated by the rear and front rotors. It reaches zero when the maximum reference altitude is reached and the flight will start the forward mode operation as shown in Figure 3(b). The gravitational force is counteracted by the thrust produced by all four rotors and the height of the vehicle is kept invariant. At the end of this mode, the pitch angle will gradually increase until it reaches $90^{\circ}$, decreasing the altitude. Then the flight will hover for a few instants and will land.

Transition is the intermediate mode of VTOL flight simulation. It is a challenging task for the UAV to alter its flight from horizontal to vertical and vice-versa. The tilt angle of the rotor changes in steps of $15^{\circ}$ interval. At each tilt angle, there will be different value thrust, velocity, lift and pitching moment as shown in Figure 5(a). Figure 5(b) shows the path traced by UAV during its entire flight. Robust Control Toolbox in MATLAB/SIMULINK is used to design an H-infinity robust controller for quad tiltrotor UAV using the Glover-McFarlane loop shaping algorithms. This robust control method is analogous to bode plots. The controller is designed using optimal H-infinity loop shaping. Maximum and minimum input-output gains are plotted using Sigma as a function of frequency [27, 28].

The designed UAV model has single input which controls two extremely coupled outputs. Figure 6(a) shows a closed-loop response of the model that eliminates the resonant pole pair with a thirdorder critically-damped filter. The graphs of the step responses of the closed-loop system $\mathrm{T}$ are plotted in Frequency for this filter is selected experimentally from Figure 6(b) which is the fastest value that overcomes the gradient reversal in the pilot experienced force. Weighting functions are selected by setting the crossover frequency equal to the target closed-loop bandwidth and then some integral weighting function is added to give a greater slope. This method will result in low gain at high frequency and high gain at low frequency, resulting in good disturbance/noise rejection. 


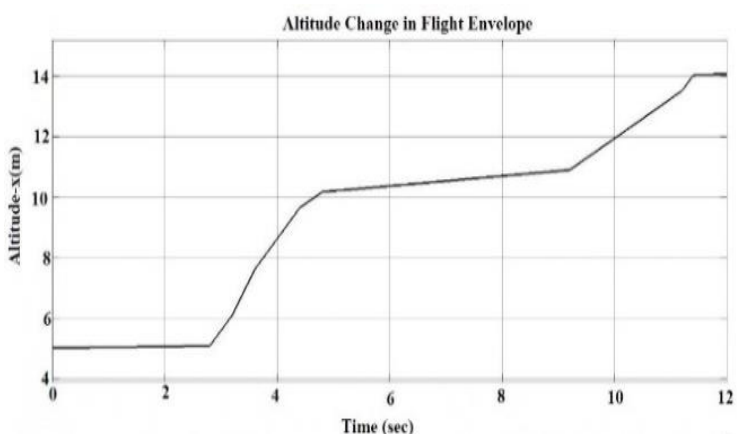

(a)

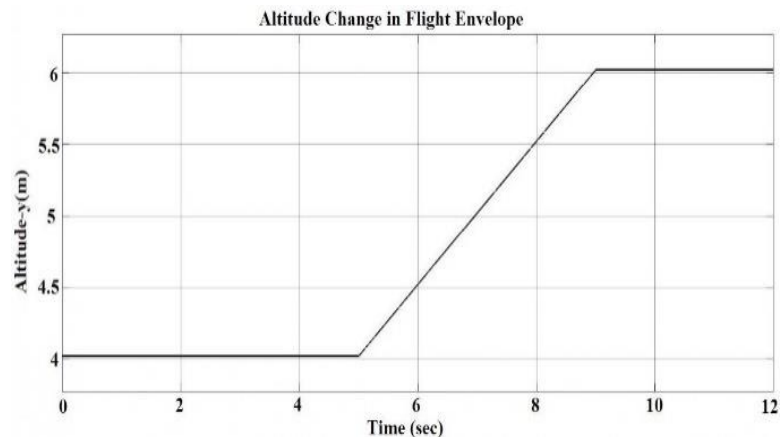

(b)

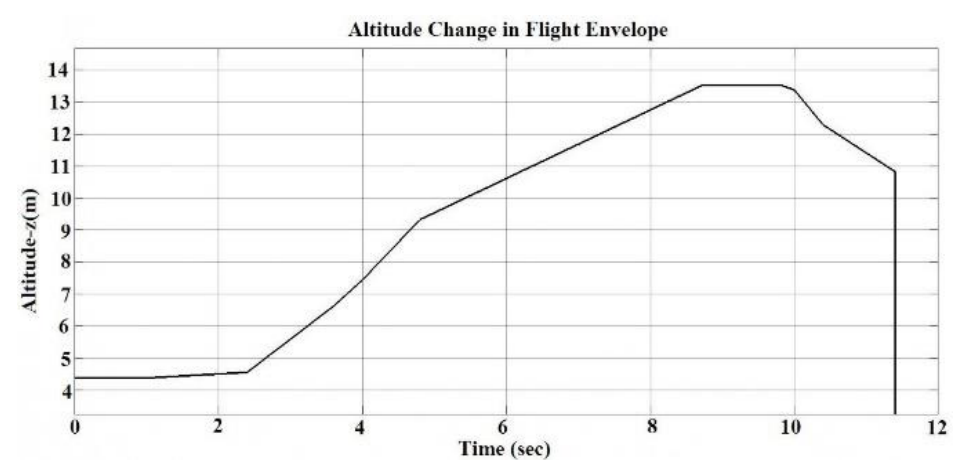

(c)

Figure 4. Change in altitude during entire flight (a) Change in $\mathrm{x}(\mathrm{m}),(\mathrm{b})$ Change in $\mathrm{y}(\mathrm{m})$, (c) Change in $\mathrm{z}(\mathrm{m})$

The design of robust $\mathrm{H}$-infinity controller $K$ is implemented such that the target-loop shape $L$ is supposed to match with the open-loop gain $P(s) . K(s)$. Comparison of the plot of $L$ and its singular values is shown in Figure 6(b). Robust stability and performance bounds of the designed controller are shown in Figure 7(a). It is evident that the system has robust stability as the gain of the target loop shape $G_{d}$ is less than $0 \mathrm{~dB}$ at high frequency.

The controller also shows robust performance because the target loop shape $L$ is having gain more than $0 \mathrm{~dB}$ at low frequency giving good disturbance rejection and high accuracy. The designed controller $K$ has improved performance. The two responses of the systems are almost identical. Hence, the designed loop shaping controller $K$ as shown in Figure 7(b), performs well under disturbance and could be used for realworld implementations.

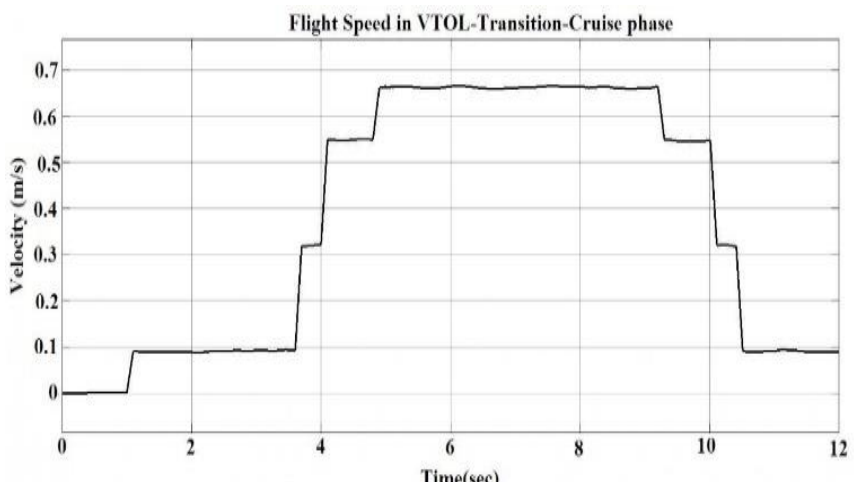

(a)

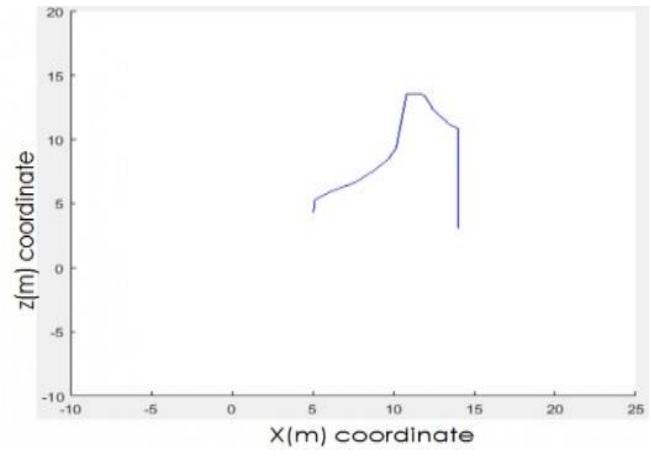

(b)

Figure 5. (a) Flight speed during the entire flight, (b) The flight path during the entire flight 

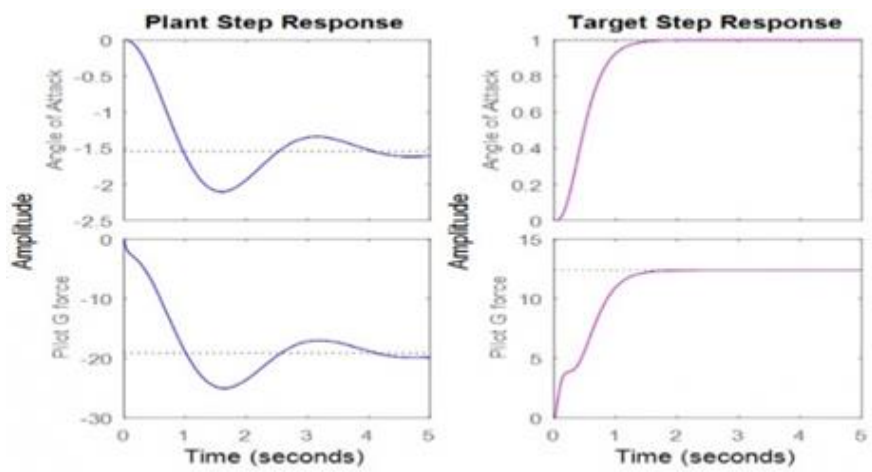

(a)

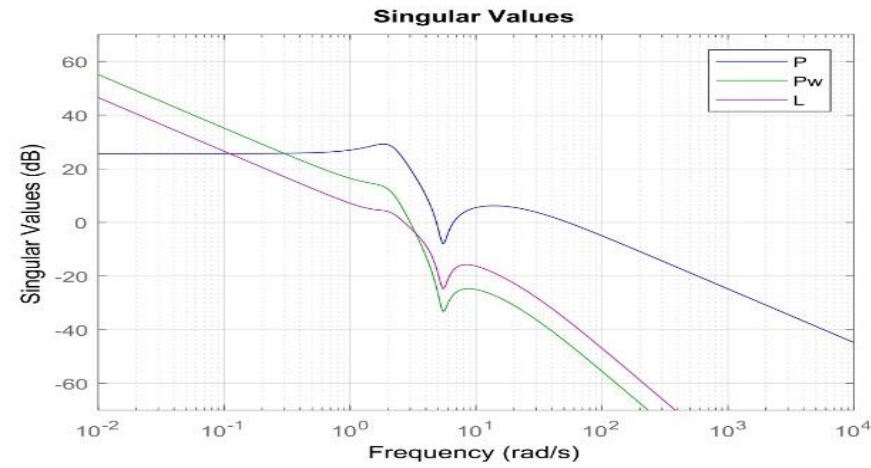

(b)

Figure 6. (a) Plant step response and target step response, (b) Plot of Singular value of plant, weighting function and Loop transfer function

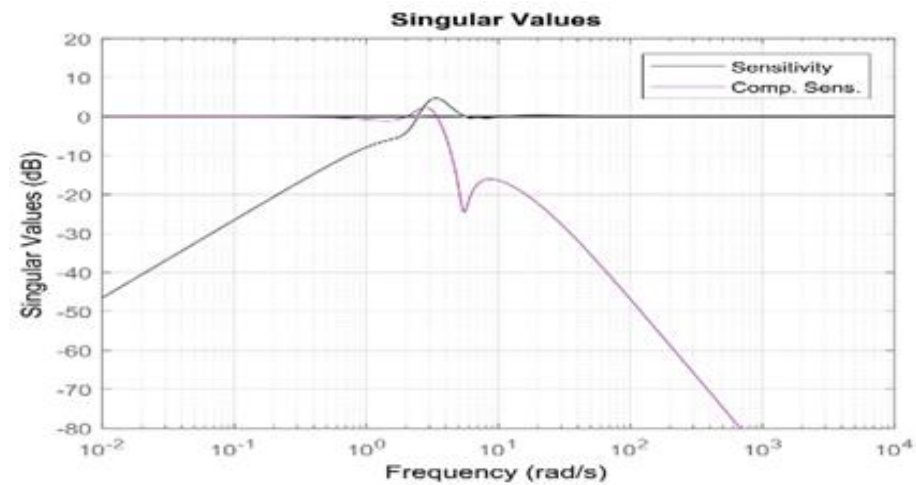

(a)

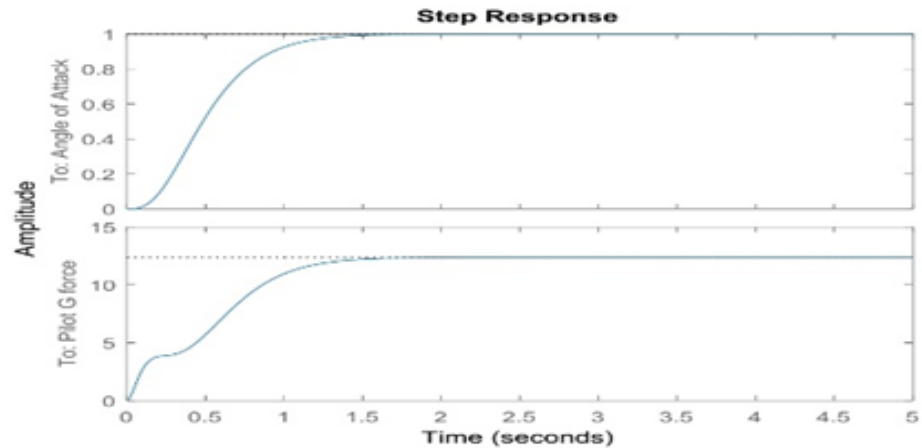

(b)

Figure 7. (a) Singular value plot of Sensitivity and complementary sensitivity functions, (b) Step response with the controller 
The system performance can be evaluated by analyzing the maximum singular value of the sensitivity function and complementary sensitivity function in Figure 7(a). The necessary condition for the system to exhibit robust performance and stability is that the sensitivity function should be maintained as small as possible at low frequencies. At higher frequencies, the control effort should be made small. From the above figures, it can be seen that UAV performs attitude stabilization accurately despite its complex, fast and highly non-linear dynamics. Figures show a slight deviation from the reference values. However, these small changes will not effect the stability of the UAV.

\section{CONCLUSION}

The 6 DoF non-linear dynamic model of the quad tiltrotor UAV is developed using MATLAB/SIMULINK. This includes aerodynamics, motor dynamics, propulsion, mass, inertia and environment modeling. Linearization of nonlinear model around the specified trim condition is performed using the MATLAB/SIMULINK Control Design Toolbox. The design technique used for the H-infinity robust controller considers the uncertainties of the model parameters and the disturbances which act upon the system. The simulation results show that the robust $\mathrm{H}$-infinity controller achieves good performance, robustness in terms of sensitivity and provides high stability and high disturbance rejection. It has been observed that the proposed method verifies the validity of the robust H-infinity loop shaping technique. The future work includes the implementation of the model into the open-source code Flight Gear for a realtime simulation to check the tracking performance.

\section{REFERENCES}

[1] M. R.AL-Obaidi., et al., "Improvement in energy conversion for unmanned aerial vehicle charging pad," Indonesian Journal of Electrical Engineering and Computer Science, vol. 17, pp. 767-773, 2020.

[2] V. E. Ömürlü., et al., "A stationary, variable DOF flight control system for an unmanned quadrocopter," Turkish Journal of Electrical Engineering \& Computer Sciences, vol. 19, pp. 891-899, 2011.

[3] P. Castillo., et al., "Modelling and control of mini-flying machines," Springer Science \& Business Media, USA, 2005.

[4] A. Al., et al., "An angle speed and thrust relationship of the quadcopter rotor," Indonesian Journal of Electrical Engineering and Computer Science, vol. 13, pp. 469-474, 2019.

[5] E. Çetinsoy., et al., "Design and development of a tilt-wing UAV," Turkish Journal of Electrical Engineering \& Computer Sciences, vol. 19, pp. 733-741, 2011.

[6] A. S. Saeed, et al., "A review on the platform design, dynamic modeling and control of hybrid UAVs," in International Conference on Unmanned Aircraft Systems (ICUAS), pp. 806-815, 2015.

[7] L. Zhong., et al., "Control techniques of tilt rotor unmanned aerial vehicle systems: A review," Chinese Journal of Aeronautics, vol. 30, pp. 135-148, 2017.

[8] G. M. Hoffmann, et al., "Quadrotor helicopter flight dynamics and control: Theory and experiment," in AIAA Guidance, Navigation and Control Conference and Exhibit, pp. 1-20, 2007.

[9] A. Mokhtari., et al., "Robust feedback linearization and GH-infinity controller for a quadrotor Unmanned aerial vehicle," Journal of Electrical Engineering, vol. 57, pp. 20-27, 2006.

[10] C. M. Lin and C. H. Leng CH, "Intelligent robust control for uncertain nonlinear multivariable systems via CMAC and H-infinity technology," in IEEE International Conference on Systems, Man and Cybernetics, pp. 2327-2330, 2008.

[11] O. Aedo., et al., "Mixed controller design for an UAV flight control system," in CHILEAN Conference on Electrical, Electronics Engineering, Information and Communication Technologies (CHILECON), pp. 135-140, 2015.

[12] A. Mokhtari and A. Benallegue A, "Dynamic feedback controller of Euler angles and wind parameters estimation for a quadrotor Unmanned aerial vehicle," in IEEE International Conference on Robotics and Automation, pp. 2359-2366, 2004.

[13] Y. Zhou., et al., "Robust tracking control of uncertain MIMO nonlinear systems with application to UAVs," "IEEE/CAA Journal of Automatica Sinica", vol. 2, pp. 25-32, 2015.

[14] J. J. Dickeson., et al., "Robust LPV H-infinity gain-scheduled hover-to-cruise conversion for a tilt-wing rotorcraft in the presence of $C G$ variations," in Proceedings of the 46th IEEE Conference on Decision and Control, pp. 2773 $2778,2007$.

[15] B. Kada and Y. Ghazzawi, "Robust PID controller design for an UAV flight control system," in Proceedings of the World Congress on Engineering and Computer Science, pp. 1-6, 2011.

[16] B. Yuksek., et al., "Transition flight modeling of a fixed-wing VTOL UAV," Journal of Intelligent \& Robotic Systems, vol. 84, pp. 83-105, 2016.

[17] T. R. Yechout, "Introduction to aircraft flight mechanics," American Institute of Aeronautics and Astronautics, Inc., 2003.

[18] K. M. Kleinhesselink, "Stability and control modeling of tiltrotor aircraft," M.Sc thesis, University of Maryland, College Park, MD, USA, 2007. 
[19] F. Çakıc1, "Modeling, stability analysis and control system design of a small-sized tiltrotor UAV," M.Sc thesis, Middle East Technical University, Ankara, Turkey, 2009.

[20] F. Çakıcı and M. K. Leblebicioğlu, "Modeling and simulation of a small-sized tiltrotor UAV," Journal of Defense Modeling and Simulation: Applications, Methodology, Technology, vol. 9, pp. 335-345, 2011.

[21] F. Çakıcı and M. K. Leblebicioğlu, "Control system design of a vertical take-off and landing fixed-wing UAV," IFAC-PapersOnLine, vol. 49, pp. 267-272, 2016.

[22] G. R. Flores., et al., "Quad-tilting rotor convertible MAV: Modeling and real-time hover flight control," Journal of Intelligent \& Robotic Systems, vol. 65, pp. 457-471, 2012.

[23] S. Bagheri, "Modeling, simulation and control system design for civil unmanned aerial vehicle (UAV), M.Sc thesis, Umeå University, Umeå, Sweden, 2014.

[24] A. C. Kahvecioğlu, "Design and Manufacturing of Quad tilt rotor unmanned aerial vehicle," M.Sc thesis, Middle East Technical University, Ankara, Turkey, 2014.

[25] J. P. P. Goméz., et al., "Robust Controllers Design Strategies for Unmanned air vehicles: H-infinity," in $25^{\text {th }}$ International Congress of the Aeronautical Sciences, pp. 1-8, 2006.

[26] K. Masuda and K. Uchiyama, "Robust control design for quad tilt-wing UAV," Aerospace, vol. 5, pp. 1-20, 2018.

[27] J. C. Doyle and G. Stein, "Multivariable feedback design: Concepts for a classical/modern synthesis," IEEE Transactions on Automatic Control, vol. 26, pp. 4-16, 1981.

[28] K. Zhou and J. C. Doyle, "Essentials of Robust control," Upper Saddle River, USA: Prentice hall, 1998.

\section{BIOGRAPHIES OF AUTHORS}

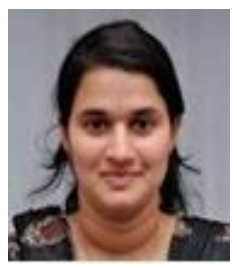

Navya Thirumaleshwar Hegde is a Research Scholar in the Department of Instrumentation and Control Engineering at Manipal Institute of Technology, MAHE, Manipal, India. Her research interests include Robust Control, Aerospace Control, System Modeling and Simulation, Autonomous Systems.

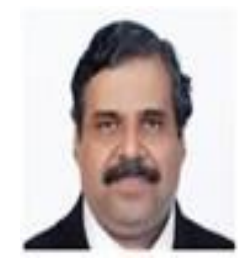

Dr. V.I.Geroge is a Professor in the department of Instrumentation and Control Engineering at Manipal Institute of Technology, MAHE, Manipal, India. His research interests include Control Systems, Optimization, Robust Control and Aerospace Control.

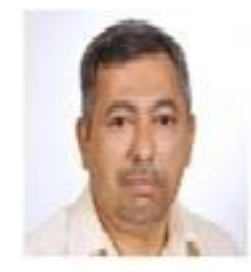

Dr. C.Gurudas Nayak is an Associate Professor - Senior Scale in the department of Instrumentation and Control Engineering at Manipal Institute of Technology, MAHE, Manipal, India. His research interests include Instrumentation and Control Engineering, Telecommunication Engineering and Biomedical Engineering.

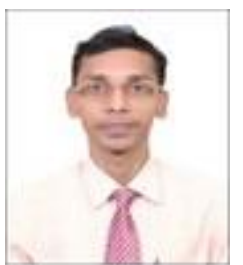

Kamlesh Kumar is an Assistant Professor - Senior Scale, In-charge of Avionics and Navigation Laboratory, Faculty Advisor at AeroMIT, Coordinator at Centre for Avionics in the Department of Aeronautical and Automobile Engineering at Manipal Institute of Technology, MAHE, Manipal, India. His research interests include Avionics and Navigation Systems, Nonlinear Control Systems Design, System Modeling and Simulation, Autonomous Systems, Missile and Mobile Robotics Guidance. 